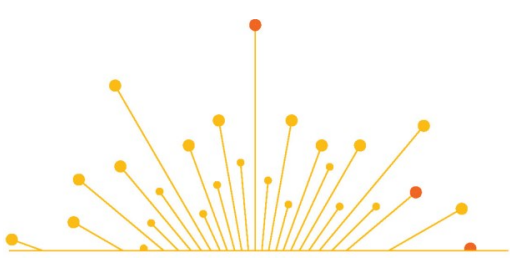

SCIENCE TABLE

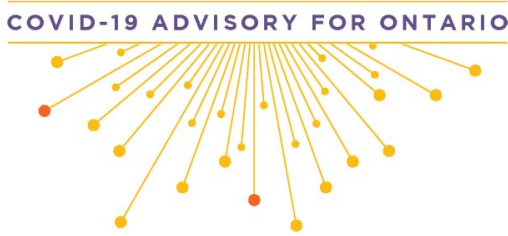

Version 1.0

Published: April 20, 2021

Citation: Fighting COVID-19 in Ontario: The Way Forward. Ontario COVID-19 Science Advisory Table. 2021;1(23). https:// doi.org/10.47326/ocsat.2021.02.23.1.0

About Us: The Ontario COVID-19 Science Advisory Table is a group of scientific experts and health system leaders who evaluate and report on emerging evidence relevant to the COVID-19 pandemic, to inform Ontario's response. Our mandate is to provide weekly summaries of relevant scientific evidence for the COVID-19 Health Coordination Table of the Province of Ontario, integrating information from existing scientific tables, Ontario's universities and agencies, and the best global evidence. The Science Table summarizes its findings for the Health Coordination Table and the public in Science Briefs.

Author Affiliations: The affiliations of the members of the Ontario COVID-19 Science Advisory Table can be found at https:// covid19-sciencetable.ca/.

Anne Hayes, Michael Hillmer, and David McKeown of the Ontario COVID-19 Science Advisory Table, and Brian Lewis, Kamil Malikov, and Bill Praamsma of the Modelling Consensus Table have recused themselves from this statement.

Correspondence to: Secretariat of the Ontario COVID-19 Science Advisory Table (info@covid19-sciencetable.ca)

Copyright: 2021 Ontario COVID-19 Science Advisory Table. This is an open access document distributed under the terms of the Creative Commons Attribution License, which permits unrestricted use, distribution, and reproduction in any medium, provided that the original work is properly cited.
STATEMENT

\section{Fighting COVID-19 in Ontario: The Way Forward}

One year into the COVID-19 pandemic, Ontario is now facing the most challenging health crisis of our time. Our case counts are at an all-time high. Our hospitals are buckling. Younger people are getting sicker. The disease is ripping through whole families. The variants of concern that now dominate COVID-19 in Ontario are, in many ways, a new pandemic. And Ontario needs stronger measures to control the pandemic.

If we want to bring cases under control, protect our health system and reopen as quickly as possible, this is the way forward.

\section{Our Principles}

Since its formation in July 2020, the Ontario COVID-19 Science Advisory Table has operated according to three principles:

1. We are guided by the most current scientific evidence.

2. We are transparent. All of our Science Briefs and presentations are publicly posted so that everyone has access to our advice and can use it.

3. We are independent. While we generally advise the provincial government of what we will say publicly, no government body or office vets or controls our scientific content or communications in any way.

\section{What Will Work}

More than one year into the COVID-19 pandemic, we know the following six things will reduce transmission, protect our health care system, and allow us to re-open safely as soon as possible:

- Essential workplaces, only: Some indoor workplaces have to remain open, but the list of what stays open must be as short as possible. This means permitting only truly essential indoor workplaces to stay open and strictly enforcing COVID19 safety measures in those places. Essential workers must wear masks at all times while working indoors, or when close to others outdoors, and must be supported in doing so.

- Paying essential workers to stay home when they are sick, exposed or need time to get vaccinated: SARS-CoV-2, the virus that causes COVID-19, spreads when people go to work sick or after having been exposed to the virus. Workers who do this, often do so because they have no choice: they must feed their families and pay their rent. Compared to other models that appear to have limited spread, the federal program is cumbersome and does not provide 
enough financial support. An emergency benefit that offers more money, is easily accessible, immediately paid and that, for the duration of the pandemic, is available to essential workers - when they are sick, when they've been exposed, need time off to get tested, or when it's their turn to get vaccinated - will help limit spread.

- Accelerating the vaccination of essential workers and those who live in hot spots: Vaccines are essential in slowing the pandemic. This means immediately allocating as many doses as possible to hotspot neighbourhoods, vulnerable populations, and essential workers. It also means accelerating the distribution and administration of vaccines overall, making it easier for at-risk groups to get vaccinated, and promoting the vaccine with more intensive and effective on-the-ground community outreach.

- Limiting mobility: This means restricting movement between regions of the province and restricting movement into the province. COVID-19 is not a single pandemic, because different regions of Ontario and Canada face distinct problems. Moving around the province risks creating new hotspots, especially because the variants of concern are so transmissible. Simply put, Ontarians need to stay in their local communities.

- Focusing on public health guidance that works: This means Ontarians can't gather indoors with people from outside their household (with the very limited exception of safe indoor work in essential workplaces). It means Ontarians can spend time with each other outdoors, distancing two metres, wearing masks, keeping hands clean.

- Keeping people safely connected: Maintaining social connections and outdoor activity are important to our overall physical and mental health. This means allowing small groups of people from different households to meet outside with masking and two-metre distancing. It means keeping playgrounds open, and clearly encouraging safe outdoor activities.

\section{What Won't Work}

- Policies that harm or neglect racialized, marginalized and other vulnerable populations will not be effective against a disease that already affects these groups disproportionately. For these reasons, pandemic policies should be examined through an equity lens to ensure that all communities benefit.

- As noted in repeated studies from around the world, inconsistent policies with no clear link to scientific evidence are ineffective in fighting COVID-19.

- Policies that discourage safe outdoor activity will not control COVID-19 and will disproportionately harm children and those who do not have access to their own greenspace, especially those living in crowded conditions.

There is no trade-off between economic, social and health priorities in the midst of a pandemic that is out of control. The fastest way back to work - and to all the other things that make life in Ontario great - is to get this disease under control as quickly as we can, together. 